\title{
Non-AIDS related malignancies 13 years after the availability of combined antiretroviral therapy
}

\author{
Roberto Manfredi \\ From $16^{\text {th }}$ International Symposium on HIV and Emerging Infectious Diseases \\ Marseille, France. 24-26 March 2010
}

\section{Background}

The introduction of combined antiretroviral therapy (cART) since the year 1996, contributed to a rapid, significant drop of frequency of all AIDS-defining opportunistic infections and some selected AIDS-related tumors (like Kaposi'sarcoma), with a consequent, remarkable reduction of both morbidity and mortality rates associated with these disease complications.

\section{Methods}

Our cohort of over 1,700 HIV-infected patients followed in two connected outpatient centres by the same physician staff were prospectively followed since the year 2000 (9 years), with special interest focused on the diagnosis, treatment and outcome of non-AIDS related malignancies.

\section{Results}

Among hematological malignancies other than nonHodgkin's lymphoma and primary central nervous system lymphoma, we observed three cases of acute myelogenic leukaemia and 4 episodes of Hodgkin's lymphoma. A greater number of solid tumors involved different organs and sites: laryngeal cancer (8 cases, with 6 episodes of papillomatous laryngeal cancer), rhinopharyngeal squamous carcinoma (4 cases), adenocarcinoma of the lung (6 cases), gastric adenocarcinoma (3 episodes), esophageal carcinoma (2 patients), prostate cancer (4 cases), bladder adnocarcinoma (3 episodes) pancreatic adenocarcinoma (2 cases), and squamos anal carcinoma ( 2 episodes). Some of these malignancies have been reported with extremely rare frequency until now (usually as single-case anecdotal reports), in particular before the cART era. The patient's age ranged from 34 to 67 years, the mortality rate of these episodes was very elevated (over 80\%), and occurred 3-41 months after diagnosis, despite appropriate surgical and/or cytotoxic chemotherapy and/or radiotherapy.

\section{Discussion}

The significantly increased life expectancy of HIV infected patients in the cART era was characterized by a proportionally increase of non-AIDS-defining tumors, which may depend on the advanced mean patients' age, their prolonged exposure to risk factor, the persisting functional immune system imbalance, and probably some direct oncogenic property of HIV itself, even when a "quantitative" recovery of CD4+ lymphocyte count has been achieved thanks to cART. The differential diagnosis of non-AIDS-associated tumors may be delayed by the low clinical suspicion, and their frequency to mimick and/or overlap infectious complications. Further epidemiological and clinical investigation is strongly warranted, to increase the awareness of this emerging phenomenon.

Published: 11 May 2010

doi:10.1186/1742-4690-7-S1-P71

Cite this article as: Manfredi: Non-AIDS related malignancies 13 years

after the availability of combined antiretroviral therapy. Retrovirology

2010 7(Suppl 1):P71.

Correspondence: Roberto.manfredi@unibo.it

Infectious Diseases, University of Bologna, S. Orsola Hospital, Bologna, Italy 